\title{
Experimental Research on beneficiation Technology for a Bulk Copper from Yunnan
}

\author{
Hui Zhang, Quanjun Liu *, Huawei Yuan, Yichao Zhang \\ ${ }^{1}$ State Key Laboratory of Complex Nonferrous Metal Resources Clean Utilization, Yunnan, China \\ Kunming University of Science and Technology, Kunming, China \\ ${ }^{2}$ Faculty of Land and Resource Engineering, Kunming University of Science and Technology, \\ Kunming, China \\ readyouzh@163.com, kmliuqj@sina.com,
}

Keywords: ore-washing, bulk flotation, regrinding, copper-sulfur separation

\begin{abstract}
In this paper, a experimental research on beneficiation technology for a sulfur-bearing bulk copper in Yunnan. Ore slime content is higher in the ore, chalcopyrite is closely associate with pyrite, the raw ore grading $0.95 \% \mathrm{Cu}$ and $17.88 \% \mathrm{~S}$.The flow sheet of raw ore-washing, stage-grinding, stage-concentration, copper-sulfur bulk flotation were adopted in the test. copper-sulfur bulk flotation while the ore was grind to fineness of $-0.074 \mathrm{~mm} 60 \%$,the obtained concentrate been cleaning again was regrinding to a fineness of $-0.074 \mathrm{~mm} 90 \%$ for copper-sulfur separation. The study examined the effect of reagent rate by flotation technique using sodium sulfide 500g/t, butyl xanthate : ammonium butyl (4:1) 60g/t, terpenic oil 20g/t, lime 2000g/t. The copper concentrate with grade of $16.12 \%$ and recovery rate $90.13 \%$,the sulfur concentrate with grade of $32.21 \%$ and recovery rate $70.72 \%$.It has some direction significance to the same sulfur-bearing bulk copper beneficiation.
\end{abstract}

\section{Introduction}

Copper is one of the metal that most widely used by human, our country is in a period of rapid economic development, In the process of full industrialization need to consume large amounts of copper resources. Copper always is scarcity resources and import a lot of every year in our country. So we need to more reasonable and efficient use of copper mine resources. The comprehensive recovery of copper and sulfur resources and effective separation is the focus in this paper.

\section{The ore properties}

We have researched on the crude ore. The analytic results of multi-element are shown in Table 1.

Table 1 Chemical multi-element analysis results of raw ore

\begin{tabular}{cccccccc}
\hline Element & $\mathrm{Cu}$ & $\mathrm{TFe}$ & $\mathrm{S}$ & $\mathrm{SiO}_{2}$ & $\mathrm{Al}_{2} \mathrm{O}_{3}$ & $\mathrm{CaO}$ & Else \\
content & 0.95 & 27.25 & 17.88 & 37.92 & 3.26 & 3.52 & 9.22
\end{tabular}

The results of multi-element analysis show that the recyclable elements in the mine are $\mathrm{Cu}, \mathrm{S}$ and Fe, in which the grade of the copper is $0.95 \%$, the grade of the iron is $27.25 \%$, the grade of the sulfur is $17.88 \%$. Most of the gangue mineral is silicate mineral, other elements have no recycling value.

\section{Process design}

Because in part of the ore pyrite and chalcopyrite closely associated, it is difficult to separate the copper and sulfur. Grinding fineness has a great impact on the flotation index and only in appropriate grinding fineness can get a better sorting index. The flow sheet of grinding test is showed in Figure 1, test results are showed in Figure 2. 


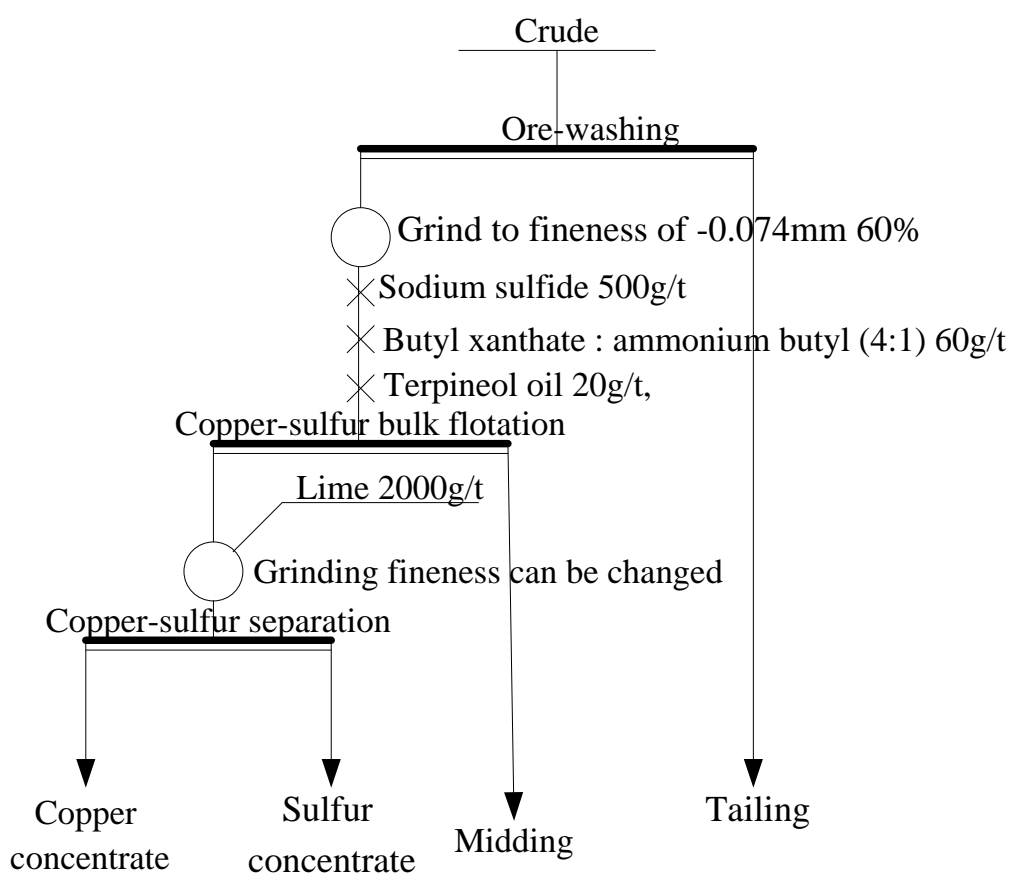

Fig1 Flow sheet of the grinding fineness test

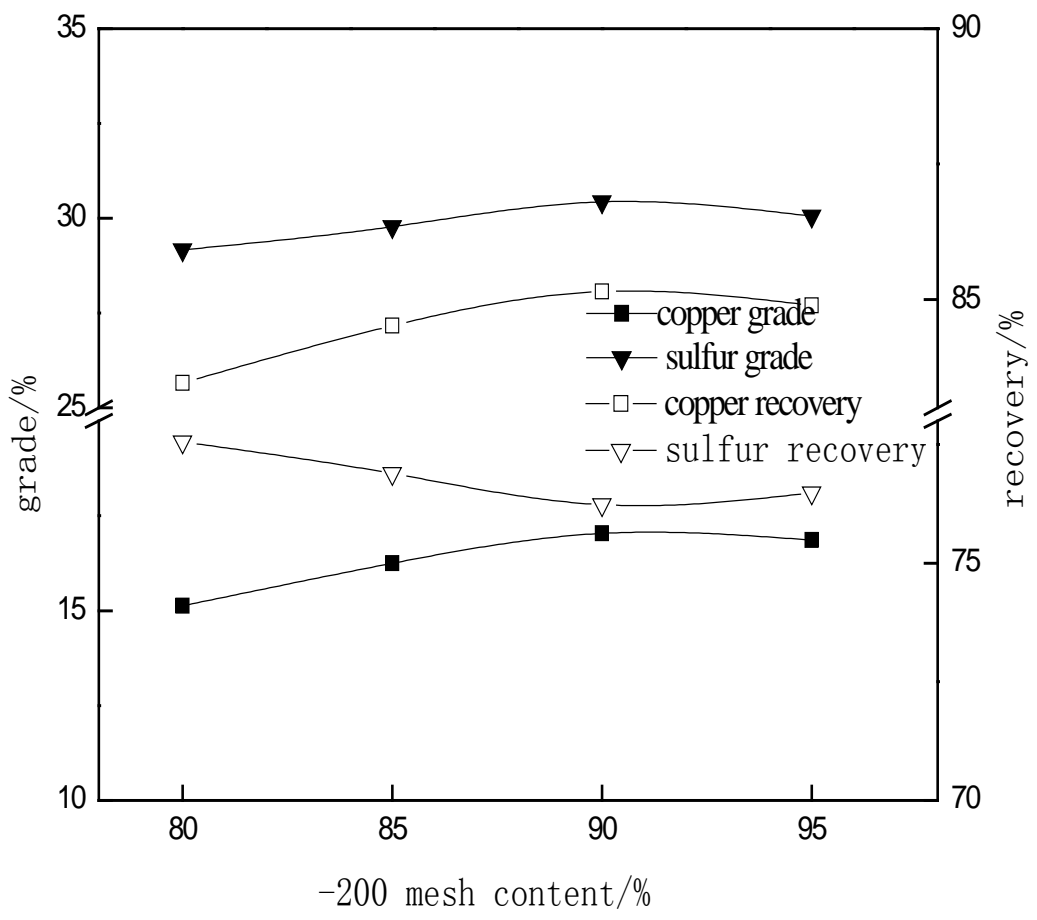

Fig 2 Results of grinding fineness test

The test results showed that with the grinding fineness increasing, the grade and recovery of copper are all increase, But the grinding fineness(-0.074mm content) reached $90 \%$, the grade and recovery of copper began to decline. Therefore, we choice the grinding fineness(-0.074mm content) is $90 \%$.

\section{Closed-circuit test}

According to the conditions of the previous tests what we have done, we conducted a small closed-circuit test, the test process shown in Figure 3, the test results shown in Table 2. 


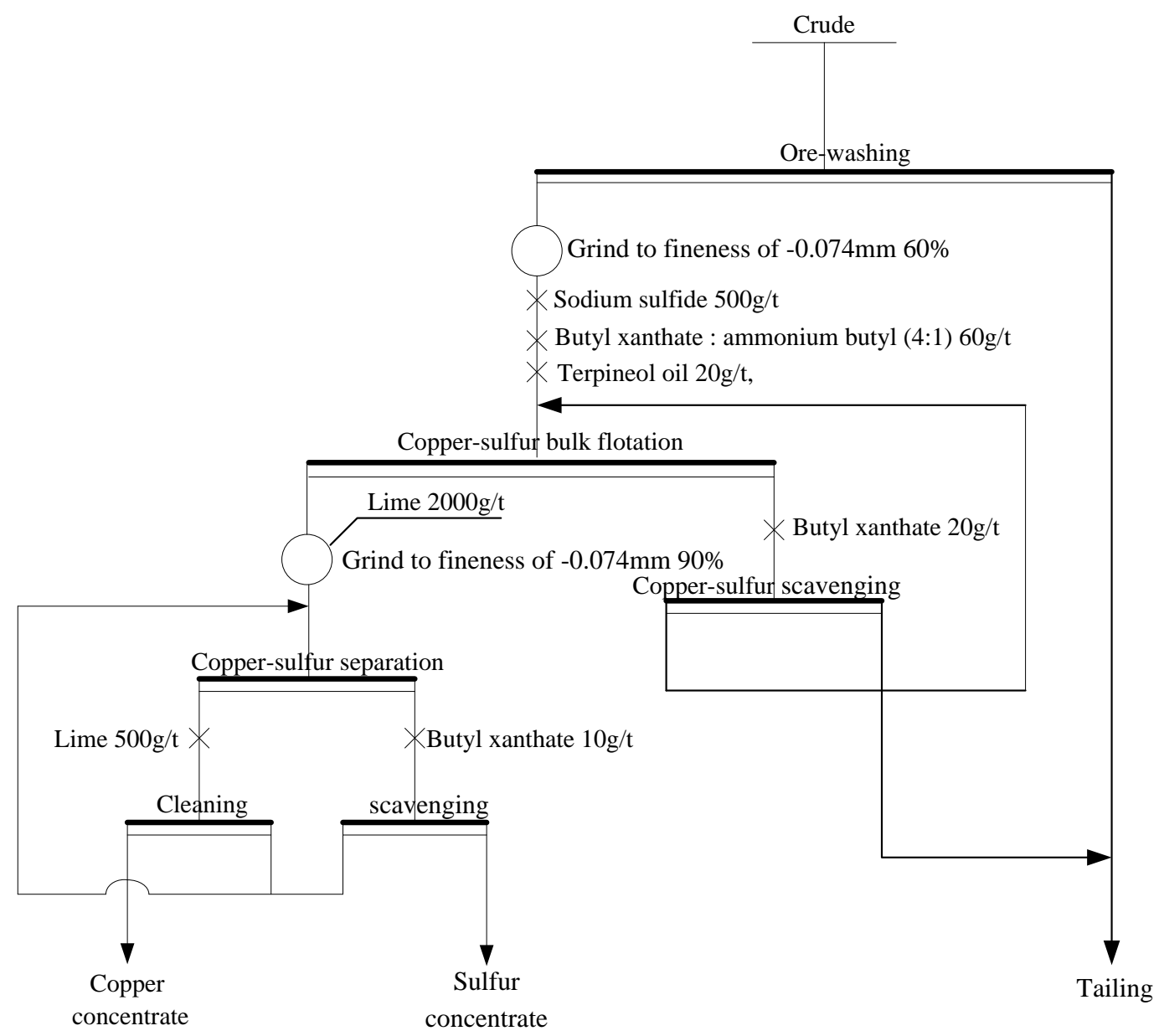

Fig 3 Closed-circuit test flowsheet

Table 2 Results of closed-circuit test

\begin{tabular}{cccccc}
\hline \multirow{2}{*}{$\begin{array}{c}\text { Product } \\
\text { name }\end{array}$} & \multirow{2}{*}{ Productivity $/ \%$} & \multicolumn{2}{c}{ Grade $/ \%$} & \multicolumn{2}{c}{ Recovery rate $/ \%$} \\
\cline { 3 - 6 } & & $\mathrm{Cu}$ & $\mathrm{S}$ & $\mathrm{Cu}$ & $\mathrm{S}$ \\
\hline Copper & 5.31 & 16.12 & 10.31 & 90.13 & 3.06 \\
Sulfur & 39.26 & 0.14 & 32.21 & 5.79 & 70.72 \\
Tailing & 55.43 & 0.07 & 8.46 & 4.08 & 26.22 \\
Crude & 100.00 & 0.95 & 17.88 & 100.00 & 100.00 \\
\hline
\end{tabular}

It can be seen table from 2, The copper concentrate with grade of $16.12 \%$ and recovery rate $90.13 \%$,the sulfur concentrate with grade of $32.21 \%$ and recovery rate $70.72 \%$.Through Closed-circuit test flow sheet achieved good experimental index.

\section{Conclusions}

1) Ore processing carried off the mud, not only saves reagents consumption and optimize the flotation conditions, to obtain a better floating index.

2) Ore mineral composition is more complex, chalcopyrite and pyrite tight symbiosis, after one-stage grinding using copper-sulfur bulk flotation. bulk concentrate with lime as a two-stage grinding inhibitors during the copper sulfur separation that reducing the energy consumption of grinding and improve recovery and grade of concentrate.

3) The copper concentrate with grade of $16.12 \%$ and recovery rate $90.13 \%$,the sulfur concentrate with grade of $32.21 \%$ and recovery rate $70.72 \%$. 


\section{References}

[1] A.L.Lu, Z.W.Sun, H.Zhang, Availability Analysis Copper Resources in China,J. Resources And Industries(2010.1)12-16

[2] D.Liu,S.M.Wen.etc, Experimental Research on the Beneficiation Technology for Separating a Refractory Mixed Copper Ore,J. Multipurpose Utilization of Mineral Resources(2010.1)11-14

[3] J.B.Qiao,S.D.Wang.etc, Study on Beneficiation Technology for a Sulfur-bearing Fe-Cu Ore from Australia,J. Mining and Metallurgy Engineering(2014,34)46-53

[4] J.C.Guo, High Sulfur Mud Oxygen Bulk Copper Ore Dressing Experiment Research,J. Hunan Nonferrous Metals(2014,29)17-19 